\title{
ProLoc-GO: Utilizing informative Gene Ontology terms for sequence-based prediction of protein subcellular localization Wen-Lin Huang ${ }^{1}$, Chun-Wei Tung ${ }^{2}$, Shih-Wen $\mathrm{Ho}^{3}$, Shiow-Fen Hwang ${ }^{1}$ and Shinn-Ying Ho*2,4
}

Address: ${ }^{1}$ Institute of Information Engineering and Computer Science, Feng Chia University, Taichung, Taiwan, ${ }^{2}$ Institute of Bioinformatics, National Chiao Tung University, Hsinchu, Taiwan, ${ }^{3}$ Department of Management Information System, Chin Min Institute of Technology, Miaoli, Taiwan and ${ }^{4}$ Department of Biological Science and Technology, National Chiao Tung University, Hsinchu, Taiwan

Email: Wen-Lin Huang - wenlinhuang2001@yahoo.com.tw; Chun-Wei Tung - wewey520@ hotmail.com; Shih-

Wen Ho - reagan@ms.chinmin.edu.tw; Shiow-Fen Hwang - sfhwang@fcu.edu.tw; Shinn-Ying Ho* - syho@mail.nctu.edu.tw

* Corresponding author

Published: I February 2008

BMC Bioinformatics 2008, 9:80 doi:10.1/86//47|-2105-9-80
Received: 25 October 2007

Accepted: I February 2008

This article is available from: http://www.biomedcentral.com/I47I-2105/9/80

(c) 2008 Huang et al; licensee BioMed Central Ltd.

This is an Open Access article distributed under the terms of the Creative Commons Attribution License (http://creativecommons.org/licenses/by/2.0), which permits unrestricted use, distribution, and reproduction in any medium, provided the original work is properly cited.

\begin{abstract}
Background: Gene Ontology (GO) annotation, which describes the function of genes and gene products across species, has recently been used to predict protein subcellular and subnuclear localization. Existing GO-based prediction methods for protein subcellular localization use the known accession numbers of query proteins to obtain their annotated GO terms. An accurate prediction method for predicting subcellular localization of novel proteins without known accession numbers, using only the input sequence, is worth developing.

Results: This study proposes an efficient sequence-based method (named ProLoc-GO) by mining informative GO terms for predicting protein subcellular localization. For each protein, BLAST is used to obtain a homology with a known accession number to the protein for retrieving the GO annotation. A large number $n$ of all annotated GO terms that have ever appeared are then obtained from a large set of training proteins. A novel genetic algorithm based method (named GOmining) combined with a classifier of support vector machine (SVM) is proposed to simultaneously identify a small number $m$ out of the $n$ GO terms as input features to SVM, where $m<<n$. The $m$ informative $G O$ terms contain the essential GO terms annotating subcellular compartments such as GO:0005634 (Nucleus), GO:0005737 (Cytoplasm) and GO:0005856 (Cytoskeleton). Two existing data sets SCLI 2 (human protein with 12 locations) and SCLI 6 (Eukaryotic proteins with 16 locations) with $<25 \%$ sequence identity are used to evaluate ProLoc-GO which has been implemented by using a single SVM classifier with the $m$ $=44$ and $m=60$ informative GO terms, respectively. ProLoc-GO using input sequences yields test accuracies of $88.1 \%$ and $83.3 \%$ for SCLI2 and SCLI6, respectively, which are significantly better than the SVM-based methods, which achieve $<35 \%$ test accuracies using amino acid composition (AAC) with acid pairs and AAC with dipedtide composition. For comparison, ProLoc-GO using known accession numbers of query proteins yields test accuracies of $90.6 \%$ and $85.7 \%$, which is also better than Hum-PLoc $(85.0 \%)$ and Euk-OET-PLoc $(83.7 \%)$ using ensemble classifiers with hybridization of $\mathrm{GO}$ terms and amphiphilic pseudo amino acid composition for SCLI2 and SCLI6, respectively.

Conclusion: The growth of Gene Ontology in size and popularity has increased the effectiveness of GO-based features. GOmining can serve as a tool for selecting informative GO terms in solving sequence-based prediction problems. The prediction system using ProLoc-GO with input sequences of query proteins for protein subcellular localization has been implemented (see Availability).
\end{abstract}




\section{Background}

Gene Ontology (GO) [1] annotation, which describes the function of genes and gene products across species, has recently been utilized to predict protein subcellular and subnuclear localization. The prediction of protein localization is important for elucidating protein functions involved in various cellular processes. Additionally, the accomplishment of the various genome sequencing projects causes the accumulation of massive amount of gene sequence information. For example, the percentage of large-scale eukaryotic proteins with subcellular locations annotated in the Swiss-Prot database increased rapidly from 52.4\% (version 49.5, released on April 18, 2006) [2] to 69.4\% (version 50.7, released Sep. 11, 2006) [3]. Meanwhile, the percentage of proteins with subcellular locations annotated in the GO database increased from $44.9 \%$ [2] to $65.5 \%$ [3]. The growth of the GO database in size and popularity increases the effectiveness of GO-based features.

Some existing computation methods in literature for predicting protein localization are described below according to the used classifiers and features.

a) Mining informative features. The prediction methods in this group focus on mining informative features consisting of GO terms [2-5], sorting signals [6,7], amino acid composition (AAC) [8-10], $k$-peptide encoding vector [7,11-14], physicochemical properties of amino acids [1517], and fusing AAC and physicochemical properties $[2,4,18,19]$.

b) Designing efficient classifiers. Most of the following prediction methods use effective classifiers based on support vector machine (SVM) $[5,10-12,14,16,17,20]$ or the $k$ nearest neighbour $(k$-NN) classifiers $[2,4,5,13,19,21]$.

c) Integrating informative features with efficient classifier. Methods in this group include pSLIP [17], ProLoc [18], Euk-OET-PLoc [2] and Hum-PLoc [4]. The pSLIP system utilizes five top-rank features of physicochemical properties according to the prediction accuracy of SVM using a single feature [17]. The ProLoc system uses SVM with automatic selection from physicochemical properties to predict protein subnuclear localization [18]. The two ensemble classifiers Euk-OET-PLoc [2] and Hum-PLoc [4] fuse many basic individual classifiers operated by the engine of $k$-NN rules, where protein sequences are represented by hybridizing the GO annotation and amphiphilic pseudo amino acid (Pse-AA) composition.

Additionally, these two efficient GO-based systems EukOET-PLoc [2] and Hum-PLoc [4] predict subcellular localization of proteins using their known accession numbers. However, they cannot work for novel proteins without known accession numbers. The GO-AA method [5], which uses GO terms of homologies retrieved by BLAST to assess protein similarity, can deal with novel proteins without known accession numbers for subnuclear localization prediction. Besides, some SVM-based methods using only the features derived from input sequences, such as ProtLock with AAC [8], Ploc with AAC and acid pairs [10], and HSLPred with AAC and dipeptide composition [11], predict subcellular localization inaccurately [4]. Therefore, this study would develop an accurate SVMbased method for predicting subcellular localization of novel proteins by using input sequences with BLAST.

The Gene Ontology provided by the GO Consortium [1] has quickly grown in size and popularity. The newest version (UniProt 52.0 released in September 2007) of GO [22] contained 29,383 terms in the three branches, molecular function, biological process and cellular component. The terms and relationships among them are represented by a directed acyclic graph in which vertices represent the GO terms, and edges represent the relationships among these terms. Genes can be annotated with GO terms creating gene associations that can be used for whole genome analyses [23].

GO annotation has been successfully used in various sequence-based applications, which can be classified into two groups. 1) The first group uses the GO terms and their corresponding structure information of GO graph, such as grouping GO terms to improve the assessment of gene set enrichment [24]; using GO with probabilistic chain graphs for protein classification $[25,26]$, and prediction of subnuclear localization [5]. 2) The second group uses GO terms only without structure information, such as predicting transcription factor DNA binding preference [27] and various predictions of subcellular and subnuclear localization $[2,4,5]$. In the second group, protein sequences are often represented as high dimensional vectors of $n$ binary features, where $n$ is the total number of terms in the complete annotation set (a component of 1 if the annotation is hit, and 0 otherwise) [28]. This representation is valuable in well-known vector space clustering algorithms such as $k$-NN $[2,4,13,19,21]$ and fuzzy $k$-NN $[13,29,30]$. However, because $n$ is often large, and each gene product is generally annotated by few GO terms, the vectors became long and sparse, making the clustering rather problematic [28].

This study proposes an efficient method, named GOmining, based on an intelligent genetic algorithm (IGA) $[31,32]$ incorporating an SVM classifier to simultaneously identify a small number $m$ out of a large number $n$ of GO terms as input features, where $m<<n$. Some GO annotations corresponding to subcellular compartments are called essential GO terms for subcellular localization pre- 
diction, such as GO:0005634 (Nucleus), GO:0005737 (Cytoplasm) and GO:0005856 (Cytoskeleton), shown in Table 1. These essential GO terms are regarded as domain knowledge to be included in the feature set of $m$ informative GO terms for subcellular localization prediction. A prediction method ProLoc-GO based on GOmining was implemented using the feature set of informative GO terms. This method performed well in predicting protein subcellular localization from input sequences only.

\section{Results}

\section{Data sets}

Two existing data sets SCL12 [4] and SCL16 [2] obtained from UniProtKB/Swiss-Prot database [33] were used to evaluate the proposed method ProLoc-GO. The SCL12 and SCL16 have 2041 human proteins localized in 12 human subcellular compartments and 4150 eukaryotic proteins in 16 subcellular compartments, respectively. The two data sets were operated by a culling program [34] so that those sequences had $<25 \%$ sequence identity.

The proteins in SCL12 were screened strictly using the following rules: 1) only those sequences annotated with "human" in the ID (identification) field were collected; 2) sequences annotated with ambiguous or uncertain terms, such as "potential", "probable", "probably", "maybe", or "by similarity", were excluded; 3 ) sequences annotated by two or more locations were excluded, and 4) sequences with less than 50 amino acid residues were removed [4]. The data set SCL12 was divided into two parts, SCL12L and SCL12T, with 919 and 1122 proteins, respectively.

Table I: Essential GO terms and their definitions

\begin{tabular}{|c|c|c|}
\hline Compartment & $\begin{array}{l}\text { Essential } \\
\text { GO term }\end{array}$ & Definition \\
\hline Centriole & GO:00058I4 & $\begin{array}{l}\text { A cellular organelle, found close to the nucleus in many eukaryotic cells, consisting of a small } \\
\text { cylinder with microtubular walls, } 300-500 \mathrm{~nm} \text { long and } 150-250 \mathrm{~nm} \text { in diameter. }\end{array}$ \\
\hline Cytoplasm & GO:0005737 & $\begin{array}{l}\text { All of the contents of a cell excluding the plasma membrane and nucleus, but including other } \\
\text { subcellular structures. }\end{array}$ \\
\hline Cytoskeleton & GO:0005856 & $\begin{array}{l}\text { Any of the various filamentous elements that form the internal framework of eukaryotic cells, and } \\
\text { typically remain after treatment of the cells with mild detergent to remove membrane constituents } \\
\text { and soluble components of the cytoplasm. }\end{array}$ \\
\hline Endoplasmic reticulum & GO:0005783 & $\begin{array}{l}\text { The irregular network of unit membranes, visible only by electron microscopy, that occurs in the } \\
\text { cytoplasm of many eukaryotic cells. }\end{array}$ \\
\hline Extracellular & GO:0030198 & $\begin{array}{l}\text { A process that is carried out at the cellular level which results in the formation, arrangement of } \\
\text { constituent parts, or disassembly of an extracellular matrix }\end{array}$ \\
\hline Golgi apparatus & GO:0005794 & $\begin{array}{l}\text { A compound membranous cytoplasmic organelle of eukaryotic cells, consisting of flattened, } \\
\text { ribosome-free vesicles arranged in a more or less regular stack. ... }\end{array}$ \\
\hline Lysosome & GO:0005764 & $\begin{array}{l}\text { Any of a group of related cytoplasmic, membrane bound organelles that are found in most animal } \\
\text { cells and that contain a variety of hydrolases, most of which have their maximal activities in the pH } \\
\text { range } 5-6 . \ldots\end{array}$ \\
\hline Chloroplast & GO:0009507 & $\begin{array}{l}\text { Any of the small, heterogeneous, artifactual, vesicular particles, } 50-150 \mathrm{~nm} \text { in diameter, that are } \\
\text { formed when some eukaryotic cells are homogenized and that sediment on centrifugation at } 100000 \\
\text { g. }\end{array}$ \\
\hline Microsome & GO:0005792 & $\begin{array}{l}\text { A semiautonomous, self replicating organelle that occurs in varying numbers, shapes, and sizes in the } \\
\text { cytoplasm of virtually all eukaryotic cells. It is notably the site of tissue respiration. }\end{array}$ \\
\hline Mitochondrion & GO:0005739 & $\begin{array}{l}\text { A membrane-bounded organelle of eukaryotic cells in which chromosomes are housed and } \\
\text { replicated.... }\end{array}$ \\
\hline Nucleus & GO:0005634 & $\begin{array}{l}\text { A small, membrane-bounded organelle that uses dioxygen }(\mathrm{O} 2) \text { to oxidize organic molecules; } \\
\text { contains some enzymes that produce and others that degrade hydrogen peroxide }(\mathrm{H} 2 \mathrm{O} 2) \text {. }\end{array}$ \\
\hline Peroxisome & GO:0005777 & $\begin{array}{l}\text { The membrane surrounding a cell that separates the cell from its external environment. It consists } \\
\text { of a phospholipid bilayer and associated proteins. }\end{array}$ \\
\hline Plasma membrane & GO:0005886 & $\begin{array}{l}\text { A cellular organelle, found close to the nucleus in many eukaryotic cells, consisting of a small } \\
\text { cylinder with microtubular walls, } 300-500 \mathrm{~nm} \text { long and } 150-250 \mathrm{~nm} \text { in diameter. It contains nine } \\
\text { short, parallel, peripheral microtubular fibrils, each fibril consisting of one complete microtubule } \\
\text { fused to two incomplete microtubules. }\end{array}$ \\
\hline Cell wall & GO:0005618 & $\begin{array}{l}\text { The rigid or semi-rigid envelope lying outside the cell membrane of plant, fungal, and most } \\
\text { prokaryotic cells, maintaining their shape and protecting them from osmotic lysis. }\end{array}$ \\
\hline Cyanelle & GO:0009842 & $\begin{array}{l}\text { Plastid type found in Glaucophyta having unstacked thylakoid membranes bearing phycobilisomes; } \\
\text { cyanelles are bound by a double membrane and a peptidoglycan layer. }\end{array}$ \\
\hline Vacuole & GO:0005773 & $\begin{array}{l}\text { A closed structure, found only in eukaryotic cells, that is completely surrounded by unit membrane } \\
\text { and contains liquid material. }\end{array}$ \\
\hline Plastid & GO:0009536 & $\begin{array}{l}\text { Any member of a family of organelles found in the cytoplasm of plants and some protists, which are } \\
\text { membrane-bounded and contain DNA. }\end{array}$ \\
\hline
\end{tabular}


Table 2: Data set SCLI 2. The data set SCLI 2 consists of SCLI 2L and SCLI 2T as the learning data set and testing data set, respectively. There are 12 essential $G O$ terms corresponding to subcellular compartments. The number $t$ of $(t)$ in SCLI $2 L$ represents the number of sequences which are correctly annotated by only one essential GO term.

\begin{tabular}{lllrr}
\hline Label & Compartment & Essential & Number of sequences \\
& & GO term & SCLI2L & SCLI2T \\
\hline I & Centriole & GO:00058I4 & $20(1)$ & 25 \\
2 & Cytoplasm & GO:0005737 & $155(38)$ & 377 \\
3 & Cytoskeleton & GO:0005856 & $12(6)$ & 14 \\
4 & Endoplasmic reticulum & GO:0005783 & $28(19)$ & 35 \\
5 & Extracellular & GO:0030198 & $140(0)$ & 301 \\
6 & Golgi apparatus & GO:0005794 & $33(5)$ & 42 \\
7 & Lysosome & GO:0005764 & $32(27)$ & 40 \\
8 & Microsome & GO:0005792 & $7(0)$ & 8 \\
9 & Mitochondrion & GO:0005739 & $125(111)$ & 228 \\
10 & Nucleus & GO:0005634 & $196(179)$ & 580 \\
II & Peroxisome & GO:0005777 & $18(16)$ & 23 \\
12 & Plasma membrane & GO:0005886 & $153(23)$ & 368 \\
\hline \multirow{2}{*}{ Total } & & & $919(425)$ & 1122 \\
\hline
\end{tabular}

The SCL12L set was used for training and the SCL12T was used for independent testing, as shown in Table 2[4].

The proteins of SCL16 were screened according to four criteria. The first criterion is to exclude sequences annotated with "prokaryotic", because this study focused only on eukaryotic proteins. The other three criteria were the same as criteria 2-4 for SCL12 above. Table 3 shows the num- bers of proteins within each compartment, where the SCL16 consists of two parts, SCL16L for training and SCL16T for independent testing. The sequences in the training and test data sets were obtained from the web servers of Euk-OET-PLoc [2] and Hum-PLoc [4].

\section{GO annotation}

This study applied the Gene Ontology Annotation (GOA) database [35], which includes GO annotations for nonredundant proteins from many species in the UniProtKB/ Swiss-Prot database [33]. The GOA database was downloaded directly from [36] (UniProt 45.0 released in Jan. 2007). The accession numbers of proteins are required for querying the GOA database to obtain GO terms. BLAST $[37,38]$ was used to obtain a homology with a known accession number to the protein for retrieving the GO terms. The corresponding accession numbers of all protein sequences in SCL12 and SCL16 were obtained by using BLAST with $h=1$ and $e=10^{-9}$.

Table 4 shows the GO annotation results of all proteins in the training data sets SCL12L and SCL16L. For SCL12L, the size of the complete set of all GO terms that appeared was $n=1714$ from the 919 human proteins. The smallest, largest and mean numbers of GO terms annotated for individual proteins were 0,35 and 8.3, respectively. The percentage of training proteins whose homologies were not annotated by any GO term (that is, the number of GO terms annotated is zero) was $1.31 \%$. For SCL16L, $n=2870$ GO terms were obtained from 2423 eukaryotic proteins. The smallest, largest and mean numbers of GO terms

Table 3: Data set SCLI6. The data set SCLI 6 consists of SCLI6L and SCLI6T as the learning data set and testing data set, respectively. There are 15 essential GO terms corresponding to eukaryotic subcellular compartments. Note that GO:00058I4 is not appeared in the set of $n=2870$ GO terms. The number $t$ of $(t)$ in SCLI6L represents the number of sequences which are correctly annotated by only one essential GO term.

\begin{tabular}{|c|c|c|c|c|}
\hline \multirow[t]{2}{*}{ Label } & \multirow{2}{*}{ Compartment } & \multirow{2}{*}{$\begin{array}{l}\text { Essential } \\
\text { GO term }\end{array}$} & \multicolumn{2}{|c|}{ Number of sequences } \\
\hline & & & SCLI6L & SCLI6T \\
\hline I & Centriole & GO:00058I4 & $17(0)$ & 4 \\
\hline 2 & Cytoplasm & GO:0005737 & $384(92)$ & 334 \\
\hline 3 & Cytoskeleton & GO:0005856 & $20(7)$ & 5 \\
\hline 4 & Endoplasmic reticulum & GO:0005783 & $91(83)$ & 22 \\
\hline 5 & Extracellular & GO:0030198 & $402(I)$ & 404 \\
\hline 6 & Golgi apparatus & GO:0005794 & $68(8)$ & 17 \\
\hline 7 & Lysosome & GO:0005764 & $37(32)$ & 9 \\
\hline 8 & Chloroplast & GO:0009507 & $207(192)$ & 51 \\
\hline 9 & Mitochondrion & GO:0005739 & $183(173)$ & 45 \\
\hline 10 & Nucleus & GO:0005634 & $474(395)$ & 695 \\
\hline 11 & Peroxisome & GO:0005777 & $52(38)$ & 12 \\
\hline 12 & Plasma membrane & GO:0005886 & $323(29)$ & 90 \\
\hline 13 & Cell wall & GO:00056I8 & $20(16)$ & 5 \\
\hline 14 & Cyanelle & GO:0009842 & $78(65)$ & 19 \\
\hline 15 & Vacuole & GO:0005773 & $36(30)$ & 8 \\
\hline 16 & Plastid & GO:0009536 & $31(1)$ & 7 \\
\hline Total & & & $2423(1162)$ & 1727 \\
\hline
\end{tabular}


Table 4: Results of GO annotation for all sequences in SCLI 2L and SCLI6

\begin{tabular}{|c|c|c|c|c|c|c|c|}
\hline \multirow[t]{2}{*}{ Data set } & \multirow[t]{2}{*}{ Total GO terms $n$} & \multicolumn{3}{|c|}{ Number of GO terms } & \multicolumn{3}{|c|}{ Number of sequences annotated by g essential GO terms } \\
\hline & & Smallest & Largest & Mean & $g=0$ & $g=1$ & $g>1$ \\
\hline SCLI2L & 1714 & 0 & 35 & 8.3 & 404 & 453 & 62 \\
\hline SCLI6L & 2870 & 0 & 50 & 7.7 & 1025 & 1247 & 151 \\
\hline
\end{tabular}

annotated were 0,50 and 7.7 , respectively. The percentage of training proteins whose homologies were not annotated was $3.96 \%$. The proteins annotated by GO are often represented as an $n$-dimensional binary feature vector, where the attribute value is 1 if the corresponding GO term is annotated, and 0 otherwise.

To know the prediction performance according to only the essential GO terms annotated, we calculated the numbers of sequences annotated by $g$ essential GO terms. Table 4 shows that 453 out of 919 (49.3\%) sequences are annotated by only one essential GO term $(g=1)$ for SCL12L, where 425 sequences are correctly annotated and 28 sequences are incorrectly annotated. The other 466 sequences annotated by zero $(g=0)$ or more than one $(g$ $>1$ ) essential GO term can not be effectively predicted. Table 2 lists the numbers of sequences which are correctly annotated by only one essential GO term for every compartment. The two GO terms, GO:0005634 (Nucleus) and GO:0005739 (Mitochondrion), made a great contribution to the prediction accuracy of $46.2 \%(=425 / 919)$, which correctly annotate a large number of sequences, 179 and 111, respectively.

As for SCL16L, the number of sequences annotated by only one essential GO term is 1247 out of 2423 (51.5\%). Table 3 lists the numbers of sequences which are correctly annotated by only one essential GO term for every compartment. Only $48.0 \%$ (= 1162/2423) of the sequences with known accession numbers can be correctly predicted by using only the annotation of essential GO terms. According to Table 3, the three essential GO terms, GO:0005634 (Nucleus, 395 out of 474), GO:0009507 (Chloroplast, 192 out of 207) and GO:0005739 (Mitochondrion, 173 out of 183), made a great contribution to prediction accuracy.

The analytic results reveal that it is not sufficient to use only essential GO terms for accurately predicting protein subcellular localization. However, the essential GO terms play an important role in designing GO-based prediction methods.

Table 5: The $m=44$ informative GO terms by applying GOmining to SCLI $2 L$. The GO terms in bold style are essential GO terms.

\begin{tabular}{|c|c|c|c|c|c|c|c|}
\hline Rank by MED & GO term & Branch & MED & Rank by MED & GO term & Branch & MED \\
\hline I & GO:0005634 & C & 390.1 & 23 & GO:0007218 & B & 57.1 \\
\hline 2 & GO:0005739 & C & 350.3 & 24 & GO:0042742 & B & 56.3 \\
\hline 3 & GO:0016021 & C & 297.6 & 25 & GO:00058I5 & C & 56.3 \\
\hline 4 & GO:0005576 & C & 136.6 & 26 & GO:0005319 & $M$ & 55.2 \\
\hline 5 & GO:0008285 & B & 72.8 & 27 & GO:0020037 & $M$ & 54.7 \\
\hline 6 & GO:00058I4 & C & 70.2 & 28 & GO:0005792 & C & 50.8 \\
\hline 7 & GO:0050909 & B & 69.3 & 29 & GO:0005856 & C & 43.0 \\
\hline 8 & GO:0008633 & B & 69.3 & 30 & GO:00052I5 & M & 42.5 \\
\hline 9 & GO:0009396 & B & 67.4 & 31 & GO:0005764 & C & 41.7 \\
\hline 10 & GO:0030198 & B & 66.9 & 32 & GO:0016757 & $M$ & 39.5 \\
\hline 11 & GO:003 I 227 & C & 66.7 & 33 & GO:0005737 & C & 37.3 \\
\hline 12 & GO:0006888* & B & 66.1 & 34 & GO:0005886 & C & 36.0 \\
\hline 13 & GO:0005859 & C & 65.4 & 35 & GO:0050896 & B & 33.6 \\
\hline 14 & GO:0005794 & C & 64.7 & 36 & GO:0005813 & C & 30.6 \\
\hline 15 & GO:0009596 & B & 64.1 & 37 & GO:0005578 & C & 28.8 \\
\hline 16 & GO:000642I & B & 63.7 & 38 & GO:00056I5 & C & 28.4 \\
\hline 17 & GO:000694I & B & 63.7 & 39 & GO:0007I65 & B & 22.7 \\
\hline 18 & GO:0005622 & C & 63.0 & 40 & GO:0006886 & B & 20.1 \\
\hline 19 & GO:0004356 & $M$ & 62.6 & 41 & GO:0030662 & C & 19.9 \\
\hline 20 & GO:0008484 & $M$ & 62.6 & 42 & GO:0005216 & $M$ & 9.7 \\
\hline 21 & GO:0017119 & C & 62.1 & 43 & GO:0005777 & C & 3.8 \\
\hline 22 & GO:0006879 & B & 58.9 & 44 & GO:0005783 & C & 1.2 \\
\hline
\end{tabular}


Table 6: The $m=60$ informative GO terms by applying GOmining to SCLI6L. The GO terms in bold style are essential GO terms.

\begin{tabular}{|c|c|c|c|c|c|c|c|}
\hline Rank by MED & GO term & Branch & MED & Rank by MED & GO term & Branch & MED \\
\hline 1 & GO:0005634 & C & 331.7 & 31 & GO:0005525 & $M$ & 56.5 \\
\hline 2 & GO:0009507 & C & 244.2 & 32 & GO:0005789 & C & 55.6 \\
\hline 3 & GO:0016020 & C & 148.1 & 33 & GO:0004725 & $M$ & 55.6 \\
\hline 4 & GO:0005739 & C & 147.8 & 34 & GO:0008270 & $M$ & 54.6 \\
\hline 5 & GO:0001844 & B & 70.7 & 35 & GO:001503I & $B$ & 54.1 \\
\hline 6 & GO:00052I2 & $M$ & 70.5 & 36 & GO:00058I3 & $\mathrm{C}$ & 52.2 \\
\hline 7 & GO:0005886 & C & 68.5 & 37 & GO:0005524 & $M$ & 51.8 \\
\hline 8 & GO:0006094 & B & 67.9 & 38 & GO:005I536 & $M$ & 51.8 \\
\hline 9 & GO:004526I & C & 67.5 & 39 & GO:0016702 & $M$ & 51.1 \\
\hline 10 & GO:0009536 & C & 66.6 & 40 & GO:0005887 & C & 48.4 \\
\hline 11 & GO:00070I0 & B & 65.8 & 41 & GO:0005905 & C & 46.1 \\
\hline 12 & GO:0030I98 & B & 65.4 & 42 & GO:0005794 & $\mathrm{C}$ & 43.6 \\
\hline 13 & GO:0009626 & B & 65.4 & 43 & GO:0005622 & C & 43.4 \\
\hline 14 & GO:0005047 & $M$ & 64.6 & 44 & GO:0005759 & C & 43.1 \\
\hline 15 & GO:0017134 & $M$ & 64.1 & 45 & GO:0005856 & C & 42.1 \\
\hline 16 & GO:0000287 & $M$ & 63.3 & 46 & GO:0016757 & $M$ & 41.7 \\
\hline 17 & GO:0006888 & B & 61.7 & 47 & GO:00056I 8 & C & 41.3 \\
\hline 18 & GO:0030234 & $M$ & 61.7 & 48 & GO:0005783 & C & 35.7 \\
\hline 19 & GO:0007323 & B & 61.0 & 49 & GO:0005773 & C & 31.2 \\
\hline 20 & GO:0008083 & $M$ & 60.8 & 50 & GO:0009842 & C & 27.2 \\
\hline 21 & GO:000452I & $M$ & 60.3 & 51 & GO:00068II & B & 25.3 \\
\hline 22 & GO:0003723 & $M$ & 60.2 & 52 & GO:0006350 & B & 24.3 \\
\hline 23 & GO:00095।4 & C & 59.9 & 53 & GO:0016740 & $M$ & 23.1 \\
\hline 24 & GO:0020015 & $\mathrm{C}$ & 59.4 & 54 & GO:0005737 & $\mathrm{C}$ & 20.2 \\
\hline 25 & GO:0000922 & C & 59.0 & 55 & GO:0005829 & C & 19.6 \\
\hline 26 & GO:000568I & C & 58.9 & 56 & GO:0016798 & $M$ & 15.5 \\
\hline 27 & GO:0030149 & $\mathrm{B}$ & 57.9 & 57 & GO:0007I86 & $B$ & 14.8 \\
\hline 28 & GO:0000917 & B & 56.9 & 58 & GO:0019843 & $M$ & 13.0 \\
\hline 29 & GO:0009405 & B & 56.8 & 59 & GO:0005764 & C & 11.8 \\
\hline 30 & GO:0005615 & C & 56.5 & 60 & GO:0005777 & $\mathrm{C}$ & 10.2 \\
\hline
\end{tabular}

\section{Selected informative GO terms}

Selecting a set of $m$ informative GO terms out of $n$ candidate GO terms is a combinatorial optimization problem $\mathrm{C}(n, m)$, which can be solved by using the intelligent genetic algorithm with an inheritance mechanism (IGA) $[31,32]$. IGA can efficiently search for the solution $S_{\mathrm{r}+1}$ to $\mathrm{C}(n, r+1)$ by inheriting a good solution $S_{\mathrm{r}}$ to $\mathrm{C}(n, r)$. This study proposes an efficient algorithm based on IGA, called GOmining, to identify a small set of $m$ informative GO terms including the essential GO terms as features to SVM. The GOmining algorithm incorporates LIBSVM [39] using series of binary classifiers. GOmining aims to maximize the training accuracy of prediction using 10-fold cross-validation (10-CV) when identifying the $m$ informative GO terms.

The SVM classifier based on the selected informative GO terms as features is called SVM-IGO. To evaluate a candidate set of $r$ informative GO terms accompanied with the SVM parameters, the prediction accuracy of 10-CV serves as a fitness function of IGA. Figure 1 shows the results of SVM-IGO from $r=40,41, \ldots, 70$. Table 5 lists the $m=44$ informative GO terms for SCL12L obtained from the highest accuracy of $89.8 \%(r=44)$, where the SVM parameters $(C, \gamma)=\left(2^{3}, 2^{-4}\right)$. Table 6 lists the $m=60$ informative GO terms for SCL16L, where the highest accuracy was $86.5 \%$, and $(C, \gamma)=\left(2^{5}, 2^{-3}\right)$.

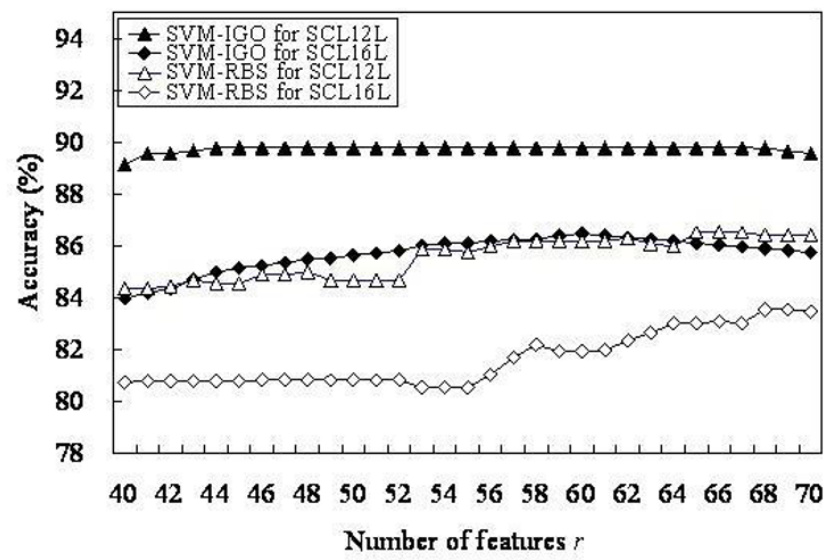

Figure I

Training accuracies of SVM-IGO and SVM-RBS performed by using SVM with a number $r$ of selected informative GO terms. 
The orthogonal experimental design with orthogonal array and factor analysis used in IGA is an efficient method for simultaneously examining the individual effect of several factors on the evaluative function $[40,41]$. The factors are the parameters (GO terms) that manipulate the evaluation function, and a setting of a parameter is regarded as a level of the factor. In this study, the two levels of one factor are the inclusion and exclusion of the ith GO term in the feature selection using IGA. The factor analysis can quantify the effects of individual factors on the evaluation function, rank the most effective factors and determine the best level for each factor to optimize the evaluation function. The most effective factor has the largest main effect difference (MED). Tables 5 and 6 show that the essential GO term GO:0005634 (Nucleus) having the largest values of MED is the most effective feature of discrimination. The only essential GO term GO:0030198 (Extracellular matrix organization and biogenesis) belongs to biologic process branch and the other essential GO terms belong to cellular component branch. The abbreviations $\mathrm{M}, \mathrm{B}$ and $\mathrm{C}$ represent the three branches molecular function, biological process, and cellular component, respectively.

\section{Evaluation of feature selection}

SVM-IGO was implemented by using the $m$ informative GO terms and the SVM classifier using $(C, \gamma)=\left(2^{3}, 2^{-4}\right)$ and $(C, \gamma)=\left(2^{5}, 2^{-3}\right)$ for SCL12L and SCL16L, respectively. To evaluate the effectiveness of SVM-IGO, four additional classifiers were implemented for comparison. Three classifiers SVM-GO, $k$-NN-GO and fuzzy $k$-NN-GO in order based on SVM, $k$-NN and fuzzy $k$-NN were implemented by using all the $n$ GO terms as features without GO term selection. The classifier SVM-RBS used SVM with a subset of $n$ GO terms selected by the rank-based selection (RBS) method [17,42]. The best values of parameters $C$ and $\gamma$ determined using a step-wise approach were employed to the SVM-based methods SVM-GO and SVM-RBS, where $\gamma$ $\in\left\{2^{-7}, 2^{-6}, \ldots, 2^{8}\right\}$ and $C \in\left\{2^{-7}, 2^{-6}, \ldots, 2^{8}\right\}$. The best values $(C, \gamma)=\left(2^{3}, 2^{-4}\right)$ and $\left(2^{4}, 2^{-6}\right)$ were applied to SVM-GO for SCL12L and SCL16L, respectively. As for SVM-RBS, $(C, \gamma)$ $=\left(2^{1}, 2^{-3}\right)$ and $\left(2^{2}, 2^{-2}\right)$ were used for SCL12L and SCL16L, respectively. The Methods section describes SVM-RBS, $k$ NN-GO and fuzzy $k-N N-G O$ in detail. Table 7 lists all prediction accuracies using 10-CV for both data sets SCL12L and SCL16L.

The highest accuracies of SVM-RBS are $86.5 \%$ and $83.5 \%$ using 65 and 68 selected GO terms for SCL12L and SCL16L, respectively, shown in Fig. 1. Table 7 shows that the three SVM-based classifiers (SVM-GO, SVM-RBS and SVM-IGO), with accuracies $>80 \%$, were better than the two $k$-NN based classifiers $(k-\mathrm{NN}-\mathrm{GO}$ and fuzzy $k$-NNGO), with accuracies $<75 \%$, for both data sets. SVM-IGO had the highest accuracies $89.8 \%$ and $86.5 \%$ for SCL12L and SCL16L, respectively. The GO term selection method based on GOmining was more effective than RBS and the method without selection of GO terms. Furthermore, SVM uses the selected GO terms as features, making it better than the $k$-NN classifier.

\section{Performance comparison}

The proposed ProLoc-GO method predicts the subcellular localization of an input sequence using either SVM-IGO or SVM-GO, depending on its annotation on the informative GO terms (see Methods for detail). Tables 8, 9, 10, 11 list the results of ProLoc-GO using SCL12 and SCL16. Some existing AAC-based prediction methods, such as ProtLock [8], Least Euclidean distance [9], Ploc [10] and HSLPred [11], use only the query sequence as input data for their classifiers. Hum-PLoc [4] and Euk-OET-PLoc [2] use both the sequence and its accession number as input data. For comparison with these predictors, the method ProLoc-GO was performed using the two kinds of input data separately. The first test used only the sequence and used BLAST to obtain annotated GO terms. The second test used the known accession number of proteins directly. For the accuracy on both SCL12L and SCL16L, ProLoc-GO used leave-one-out cross-validation (LOOCV) for comparison with the other methods (see Methods section).

The test accuracies for ProLoc-GO performed on the human protein data set SCL12L and SCL12T were 90.0\% and $88.1 \%$, respectively, where $\mathrm{m}=44$, and $(\mathrm{C}, \gamma)=(23$, 2-4) for SVM-IGO and SVM-GO. These results were much better than those $(<35 \%)$ of the four sequence-based prediction methods [8-11] using only input sequences, shown in Table 8. Ploc [10] had the highest test accuracy of $34.3 \%$ among the four AAC-based methods.

The Matthews correlation coefficient (MCC) $[5,12,18]$ values are usually employed while evaluating the performance on unbalanced datasets. In addition to the overall accuracy, the MCC values were also recorded due to the unbalance of numbers of proteins localized in the compartments, such as 196 of Nucleus vs. 7 of Microsome (Table 2). The MCC is defined as follows [5]:

$M_{C C}=\frac{p_{c} s_{c}-u_{c} o_{c}}{\sqrt{\left(p_{c}+u_{c}\right)\left(p_{c}+o_{c}\right)\left(s_{c}+u_{c}\right)\left(s_{c}+o_{c}\right)}}, \quad c=1,2, \ldots, N_{c^{\prime}}$

where $p_{c}$ is the number of correctly predicted proteins of the location $c, s_{c}$ is the number of correctly predicted proteins not in the location $c, u_{c}$ is the number of under-predicted proteins, $o_{c}$ is the number of over-predicted proteins, and $N_{c}$ is the number of locations. The test MCC performances of ProLoc-GO were 0.822 and 0.661 for SCL12L and SCL12T, respectively. Table 10 presents the 
Table 7: Comparison of prediction accuracy (\%) using I0-CV. Performance comparison uses prediction accuracy (\%) of I0-CV.

\begin{tabular}{lccccc}
\hline Data set & $k$-NN-GO & Fuzzy k-NN-GO & SVM-GO & SVM-RBS & SVM-IGO \\
\hline SCLI2L & 74.3 & 71.4 & 88.5 & 86.5 & 89.8 \\
SCLI6L & 66.0 & 59.3 & 84.8 & 83.5 & 86.5 \\
\hline
\end{tabular}

detailed results for individual compartments. The results of the five sequence-based methods reveal that the set of informative GO terms is more useful for protein subcellular localization than the AAC-based features.

\section{Performance of using known accession numbers}

The accession number of each protein sequence in SCL12 and SCL16 was available in querying the GOA database. For comparison with the methods $[2,4]$ based on the proteins with known accession numbers, ProLoc-GO using the known accession numbers of proteins as input data obtained test accuracies of $91.1 \%$ and $90.6 \%$ (MCC = $0.724)$ performed on SCL12L and SCL12T, respectively, where $m=56,(C, \gamma)=\left(2^{2}, 2^{-1}\right)$ for SVM-IGO and $(C, \gamma)=$ $\left(2^{2}, 2^{-4}\right)$ for SVM-GO. Hum-PLoc [4] using hybridization of GO terms and Pse-AA composition obtained training and test accuracies of $81.1 \%$ and $85.0 \%$ for SCL12L and SCL12T, respectively. The performance of ProLoc-GO using sequences or accession numbers as the input data was better than that of Hum-PLoc [4] using the ensemble classifiers with features of both sequence and accession number.

Tables 9 and 11 show the performance results of ProLocGO and Euk-OET-PLoc [2] using SCL16. ProLoc-GO using input sequences yielded test accuracies 86.6\% (MCC = $0.799)$ and $83.3 \%(\mathrm{MCC}=0.706)$ for SCL16L and SCL16T, respectively, where $m=60,(C, \gamma)=\left(2^{5}, 2^{-3}\right)$ for SVM-IGO, and $(C, \gamma)=\left(2^{4}, 2^{-6}\right)$ for SVM-GO. ProLoc-GO is significantly better than all the AAC-based methods with test accuracies smaller than 35\%. ProLoc-GO yields the test accuracies $89.0 \%$ and $85.7 \%(\mathrm{MCC}=0.710)$ for SCL16L and SCL16T, respectively, using the known accession numbers of proteins, where $m=60,(C, \gamma)=\left(2^{2}, 2^{-3}\right)$ for SVM-IGO and $(C, \gamma)=\left(2^{3}, 2^{-5}\right)$ for SVM-GO. Euk-OETPLoc [2] using the ensemble classifiers with features of both sequence and accession number obtains training and test accuracies of $81.6 \%$ and $83.7 \%$, respectively. ProLoc-GO performed better than Euk-OET-PLoc on SCL16 using either sequences or accession numbers as the input data [2].

\section{Analysis of informative GO terms}

The GOmining method identifies a feature set of $m$ effective GO terms, called informative GO terms, to design an accurate SVM-based prediction method. Table 12 shows the distribution of the $m$ informative GO terms in the GO graph. For SCL12L with $m=44$, GOmining selected 12 essential GO terms and 32 instructive GO terms. The 32 instructive GO terms consist of 7 GO terms from the molecular function branch, 14 terms from the biological process branch, and 11 terms from the cellular component branch, denoted as 7(M), 14(B) and 11(C), respectively. Analytical results reveal that all the three branches contain instructive GO terms.

Due to the high correlation among GO terms in the GO graph, the feature selection of SVM should consider simultaneously a set of informative GO terms, rather than individual GO terms. Since the essential GO terms are always included, GOmining benefits from a confined search space of candidate instructive GO terms. Considering the position relationships between instructive and essential GO terms in the GO graph, instructive GO terms belonged to one of the three classes: (a) offspring but not ancestor of some essential GO term; (b) between two essential GO terms, and (c) not offspring of any essential GO term. Of the 32 instructive GO terms, 4, 2 and 26 GO

Table 8: Comparison of prediction accuracy (\%) for SCLI 2. Prediction accuracies (\%) for using leave-one-out cross-validation (LOOCV) on SCLI 2L and independent test on SCLI2T are obtained from the paper [4]. The input data is sequence only (S) or sequence with accession number (AN).

\begin{tabular}{|c|c|c|c|c|}
\hline Method & Input data & Features & LOOCV SCLI2L & Independent test SCLI2T \\
\hline ProtLock [8] & S & AAC & 29.7 & 26.4 \\
\hline Least Euclidean distance [9] & S & AAC & 30.1 & 29.5 \\
\hline Ploc $[10]$ & $\mathrm{S}$ & $A A C$ and amino acid pairs & 30.5 & 34.3 \\
\hline HSLPred [II] & $S$ & AAC and dipeptide composition & 30.7 & 33.3 \\
\hline ProLoc-GO & S & GO terms using BLAST & 90.0 & 88.1 \\
\hline Hum-PLoc [4] & S with AN & Hybridization of GO terms and Pse-AA & 81.1 & 85.0 \\
\hline ProLoc-GO & S with AN & GO terms (No BLAST) & 91.1 & 90.6 \\
\hline
\end{tabular}


terms belonged to the classes (a), (b) and (c), respectively. The 26 GO terms consist of 7(M), 14(B) and 5(C). The GO terms near the root of the GO graphs are considered to be more generic while terms near the leaves are more specific [23]. Of the instructive GO terms, 81.2\% (26/32) were not offspring of any essential GO term. These analytical results reveal that the essential GO terms are informative enough in predicting subcellular localization, and are effective in confining the space of searching instructive GO terms. The other six instructive GO terms from the cellular component branch have more specific functions than the essential GO terms in discrimination of the subcellular localization.

Figures 2, 3, 4 illustrate some of the instructive GO terms belonging to the three classes. Three instructive GO terms were found to belong to class (a), namely SCL12L: GO:0031227 (Intrinsic to endoplasmic reticulum membrane, rank 11), GO:30662 (Coated vesicle membrane, rank 41) and GO:0017119 (Golgi transport complex, rank 21), according to Fig. 2. The two terms belonging to class (b), namely GO:0005815 (Microtubule organizing center, rank 25) and GO:0005813 (Centrosome, rank 36), were found between the essential GO terms GO:0005856 (Cytoskeleton) and GO:0005814 (Centriole), as shown in Fig. 3. According to Fig. 4, five instructive GO terms belonging to the class (c) were not offspring of essential GO terms, GO:0016021 (Integral to membrane, rank 3), GO:0005576 (Extracellular region, rank 4), GO:0005622 (intracellular, rank 18), GO:0005578 (Proteinaceous extracellular matrix, rank 37) and GO:0005615 (Extracellular space, rank 38).

The $\mathrm{m}=60$ informative GO terms for SCL16L comprises 15 essential GO terms and 45 instructive GO terms. The 45 instructive GO terms consisted of $18(\mathrm{M}), 13(\mathrm{~B})$ and 14(C). The numbers of instructive GO terms coming from each branch were not significantly different. However, the numbers of instructive GO terms belonging to the three classes (a), (b) and (c) are 9, 0 and 36, respectively, which are very different. $80 \%(36 / 45)$ of the instructive GO terms were not offspring of any essential GO term. The 9 instructive GO terms belonging to the class (a) had 5, 2 and 2 terms, respectively, as shown in Figs. 2, 3 and 4. Class (c) has five GO terms with a dot-pattern box: GO:0005622 (intracellular), GO:0005615 (Extracellular space), GO:0020015 (Glycosome), GO:0016020 (Membrane) and GO:0045261 (Proton-transporting ATP synthase complex, catalytic core F(1)), as revealed by Fig. 4.

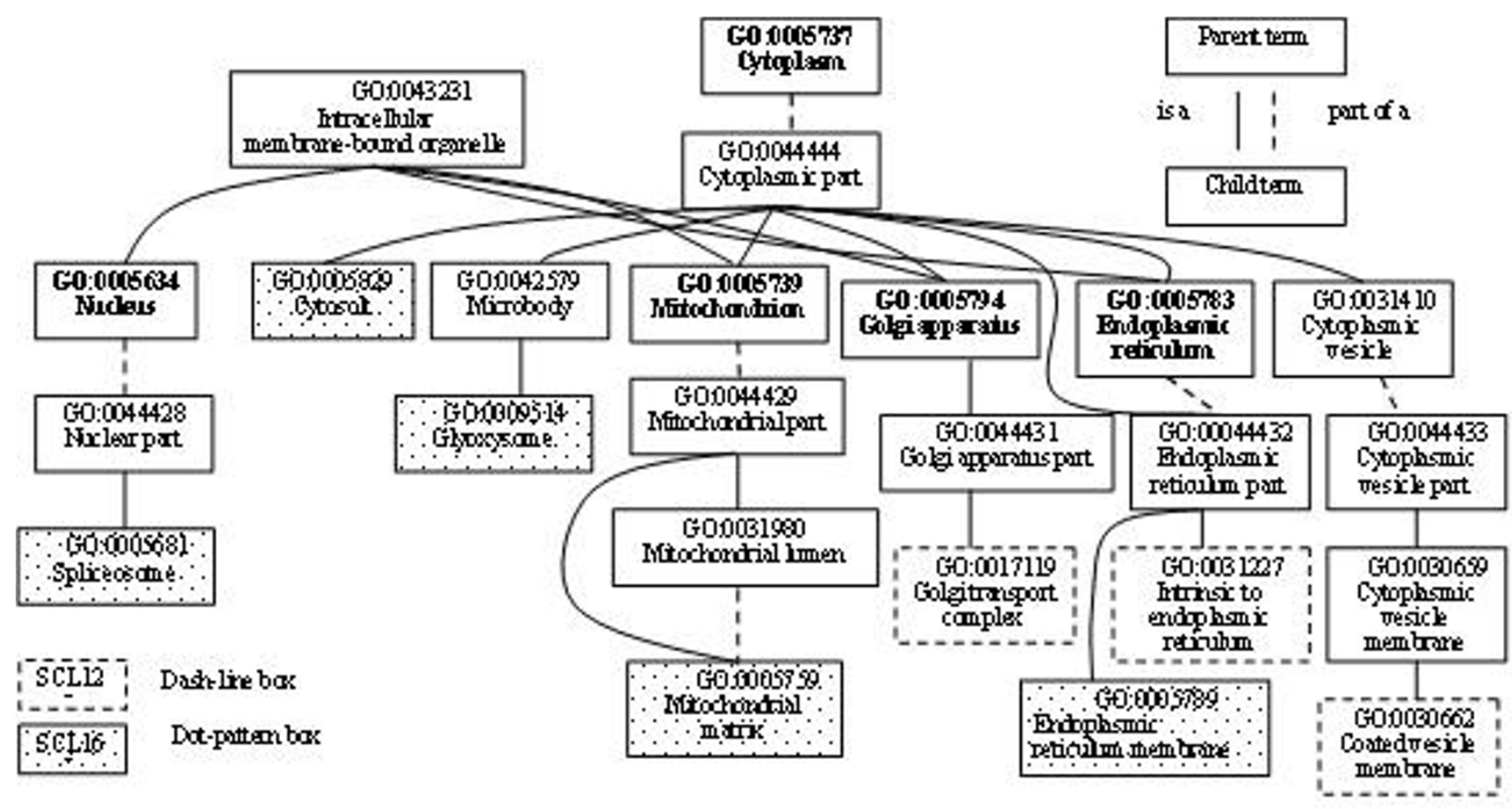

Figure 2

Some of the selected GO terms which are offspring of essential GO terms. For SCLI2L, there are three terms shown: GO:003 I 227, GO:0030662 and GO:00 I 7 I 19. For SCLI6L, five GO terms are shown: GO:00095 I4, GO:000568I, GO:0005789, GO:0005759 and GO:0005829. 


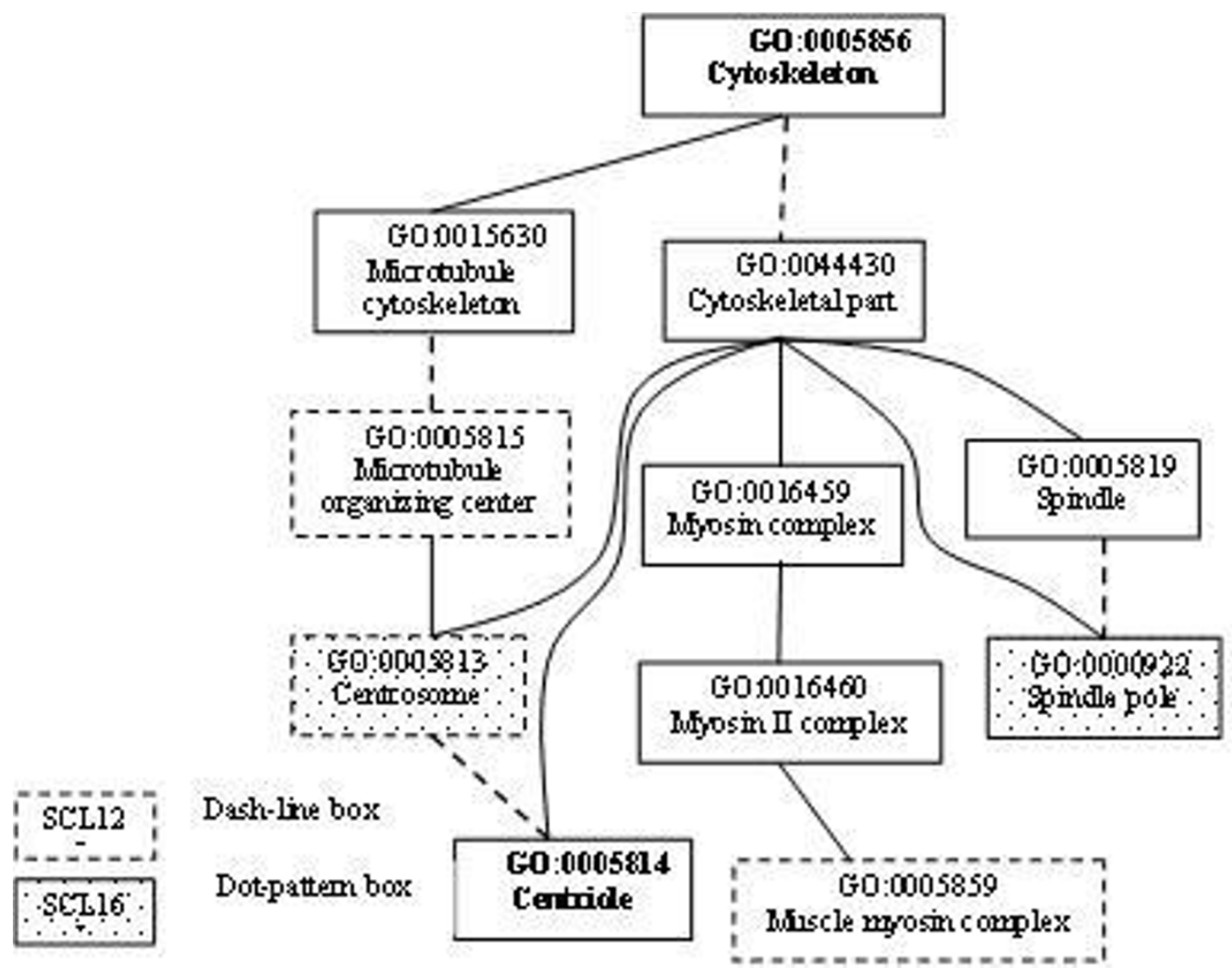

Figure 3

Some of the selected GO terms which are between two essential GO terms. For SCLI2L, the two instructive GO terms GO:00058I 5 and GO:00058I 3 are between the essential GO terms GO:0005856 and GO:00058I4. For SCLI6L, GO:00058I 3 and GO:0000922 are offspring of the essential GO term GO:0005856, belonging to the class (a). GO:00058I4 is not an essential GO term for SCLI6L.

The statistical results of instructive GO terms distributed in the three classes for both SCL12L and SCL16L reveal that the inclusion of essential GO terms can be regarded as using domain knowledge for GOmining to mine a feature set of informative GO terms. The heuristic approach (using domain knowledge) of GOmining is efficient when the GO database grows fast. Therefore, GOmining can be easily applied to other applications of sequence- based predictions using SVM with the features of informative GO terms.

\section{Discussion}

The GO database has grown in size recently, increasing the effectiveness of GO-based features. Meanwhile, the percentage of proteins with subcellular locations annotated in the GO database increased from $44.9 \%$ [2] to $65.5 \%$ [3]

Table 9: Comparison of prediction accuracy (\%) for SCLI 6. Prediction accuracies (\%) of using LOOCV on SCLI 6L and independent test on SCL I 6T are obtained from the paper [2]. The input data is sequence only (S) or sequence with accession number (AN).

\begin{tabular}{|c|c|c|c|c|}
\hline Method & Input data & Features & LOOCV SCLI6L & Independent test SCLI6T \\
\hline ProtLock [8] & $\mathrm{S}$ & AAC & 28.7 & 25.3 \\
\hline Least Euclidean distance [9] & $\mathrm{S}$ & AAC & 25.8 & 20.4 \\
\hline Ploc [10] & $\mathrm{S}$ & AAC and amino acid pairs & 35.1 & 32.8 \\
\hline HSLPred [II] & $\mathrm{S}$ & AAC and dipeptide composition & 33.1 & 34.5 \\
\hline ProLoc-GO & $\mathrm{S}$ & GO terms using BLAST & 86.6 & 83.3 \\
\hline Euk-OET-PLoc [2] & S with $A N$ & Hybridization of GO terms and Pse-AA & 81.6 & 83.7 \\
\hline ProLoc-GO & S with AN & GO terms (No BLAST) & 89.0 & 85.7 \\
\hline
\end{tabular}


Table 10: Accuracies and MCC preformed on SCLI 2

\begin{tabular}{|c|c|c|c|c|}
\hline \multirow[t]{2}{*}{ Label } & \multirow[t]{2}{*}{ Compartment } & \multirow{2}{*}{$\begin{array}{l}\text { SCLI2L } \\
\text { Sequence }\end{array}$} & \multicolumn{2}{|c|}{ SCLI2T } \\
\hline & & & Sequence & Accession no. \\
\hline 1 & Centriole & $65.0(0.803)$ & $60.0(0.670)$ & $60.0(0.774)$ \\
\hline 2 & Cytoplasm & $88.4(0.784)$ & $82.9(0.734)$ & $85.1(0.790)$ \\
\hline 3 & Cytoskeleton & $16.7(0.406)$ & $0.0(-0.002)$ & $50.0(0.314)$ \\
\hline 4 & Endoplasmic reticulum & $89.3(0.804)$ & $71.4(0.501)$ & $85.7(0.603)$ \\
\hline 5 & Extracellular & $86.4(0.87 I)$ & $76.4(0.802)$ & $79.5(0.830)$ \\
\hline 6 & Golgi apparatus & $48.5(0.630)$ & $33.3(0.328)$ & $44.4(0.364)$ \\
\hline 7 & Lysosome & $96.9(0.952)$ & $87.5(0.744)$ & $87.5(0.781)$ \\
\hline 8 & Microsome & $85.7(0.800)$ & $100.0(0.446)$ & $100.0(0.446)$ \\
\hline 9 & Mitochondrion & $99.2(0.986)$ & $97.1(0.978)$ & $100.0(0.995)$ \\
\hline 10 & Nucleus & 95.9 (0.939) & $94.3(0.930)$ & $96.9(0.952)$ \\
\hline 11 & Peroxisome & $94.4(0.943)$ & $100.0(0.912)$ & $100.0(0.912)$ \\
\hline 12 & Plasma membrane & $96.1(0.942)$ & $90.7(0.893)$ & $91.6(0.921)$ \\
\hline \multicolumn{2}{|c|}{ Overall accuracy \% (MCC) } & $90.0(0.822)$ & $88.1(0.661)$ & $90.6(0.724)$ \\
\hline
\end{tabular}

fast. It is indicated that there is a linkage in the GO annotation process between molecular function annotation and subcellular localization annotation [43]. Therefore, the GO-based prediction method for protein subcellular localization is increasingly efficient. Because the accession number of proteins is necessary for retrieving GO terms from GO databases, existing efficient GO-based systems Euk-OET-PLoc [2] and Hum-PLoc [4] directly utilize the accession numbers of proteins and a large number $n$ of GO terms annotated in a complete set where $n=9918$ for SCL12L [4] and $n=9567$ for SCL16L [2].
To predict subcellular localizations for novel proteins, ProLoc-GO uses a good homology, rather than the query protein itself, to retrieve annotated GO terms using BLAST. To use GO term features effectively, ProLoc-GO uses only a homology with annotated GO terms to reduce $n$. Thus, $n=1714$ for SCL12L and $n=2870$ for SCL16L. Furthermore, a small set of $m$ informative GO terms is selected simultaneously by GOmining. GOmining can consider internal relevant-feature correlation, instead of individual features by using an efficient global optimization method. The distribution analysis of informative GO terms in the GO graph is consistent with the properties of

Table II: Accuracies and MCC preformed on SCLI6

\begin{tabular}{|c|c|c|c|c|}
\hline \multirow[t]{2}{*}{ Label } & \multirow[t]{2}{*}{ Compartment } & \multirow{2}{*}{$\begin{array}{l}\text { SCLI6L } \\
\text { Sequence }\end{array}$} & \multicolumn{2}{|c|}{ SCLI6T } \\
\hline & & & Sequence & Accession no. \\
\hline I & Centriole & 61.1 (0.747) & $66.7(0.729)$ & $50.0(0.577)$ \\
\hline 2 & Cytoplasm & $72.9(0.706)$ & $74.6(0.676)$ & $72.8(0.659)$ \\
\hline 3 & Cytoskeleton & $20.0(0.363)$ & $0.0(-0.002)$ & $0.0(-0.003)$ \\
\hline 4 & Endoplasmic reticulum & $90.1(0.841)$ & $77.3(0.707)$ & $72.7(0.678)$ \\
\hline 5 & Extracellular & $89.6(0.778)$ & $84.4(0.738)$ & $84.9(0.796)$ \\
\hline 6 & Golgi apparatus & $63.2(0.653)$ & $41.2(0.508)$ & $41.2(0.486)$ \\
\hline 7 & Lysosome & $97.3(0.973)$ & $100.0(0.865)$ & $100.0(0.865)$ \\
\hline 8 & Chloroplast & $98.6(0.989)$ & $100.0(0.990)$ & $100.0(0.990)$ \\
\hline 9 & Mitochondrion & $99.5(0.963)$ & 91.1 (0.879) & $95.6(0.877)$ \\
\hline 10 & Nucleus & $89.0(0.913)$ & $86.3(0.865)$ & $93.5(0.924)$ \\
\hline II & Peroxisome & $98.1(0.97 I)$ & $100.0(0.960)$ & $100.0(0.925)$ \\
\hline 12 & Plasma membrane & $86.4(0.85 I)$ & $88.9(0.773)$ & $82.2(0.798)$ \\
\hline 13 & Cell wall & $75.0(0.655)$ & $60.0(0.422)$ & $80.0(0.631)$ \\
\hline 14 & Cyanelle & $88.5(0.939)$ & 94.7 (0.973) & $100.0(1.000)$ \\
\hline 15 & Vacuole & 86.1 (0.847) & $62.5(0.790)$ & $50.0(0.706)$ \\
\hline 16 & Plastid & $51.6(0.595)$ & $42.9(0.426)$ & $42.9(0.311)$ \\
\hline \multicolumn{2}{|c|}{ Overall accuracy \% (MCC) } & $86.6(0.799)$ & $83.3(0.706)$ & $85.7(0.710)$ \\
\hline
\end{tabular}


GO annotation. Additionally, ProLoc-GO using input sequences is slightly worse than using the accession numbers of proteins, with accuracies of $88.1 \%$ vs. $90.6 \%$ for SCL12T, and $83.3 \%$ vs. $85.7 \%$ for SCL16T, as shown in Tables 10 and 11.

\section{Conclusion}

Computational prediction methods from primary protein sequences are fairly economical in terms of identifying large-scale eukaryotic proteins with unknown functions. The GO annotation, which describes the function of genes and gene products across species, has been used to improve the prediction of protein subcellular localization. The accession numbers of proteins are necessary to query the GOA database to obtain GO terms. Since novel proteins have no known accession numbers, BLAST was used to obtain homologies with known accession numbers to the proteins for the retrieval of GO terms.

GO annotation has grown in size and popularity. However, few studies have explored informative GO terms from the over 20,000 annotations available at present for sequence-based prediction problems. This study proposes a genetic algorithm based method, GOmining, which combines SVM to simultaneously identify a small number $m$ out of the $n$ GO terms as features to SVM, where $m<<n$. The $m$ GO terms include the essential GO terms annotating subcellular compartments such as GO:0005634 (Nucleus), GO:0005737 (Cytoplasm) and GO:0005856 (Cytoskeleton). ProLoc-GO was evaluated using SVM with the GO-based features from two kinds of input data, sequence and known accession numbers of proteins.

ProLoc-GO yields test accuracies of $88.1 \%$ and $83.3 \%$ from SCL12 and SCL16, respectively, when using only input sequences. These results are significantly superior to those of the other SVM-based methods, which have accuracies $<35 \%$ using AAC with acid pairs, and using AAC with dipedtide composition. ProLoc-GO using known accession numbers of proteins has accuracies $90.6 \%$ and $85.7 \%$ for SCL12 and SCL16, which is also slightly better than Hum-PLoc and Euk-OET-PLoc, which have 85.0\% and $83.7 \%$, respectively.

Analysis of $m$ informative GO terms in the GO graph reveals that GOmining can consider internal relevant-feature correlation, rather than individual features, by using an efficient global optimization method. GOmining can serve as an efficient tool for mining informative GO terms for various sequence-based predictions of proteins, especially when the GO database grows fast. The prediction system using ProLoc-GO with protein sequence as input data for protein subcellular localization has been implemented (see Availability).

\section{Methods \\ Proposed GOmining algorithm}

An efficient genetic-algorithm-based method, called GOmining, is proposed for selecting informative GO terms. GOmining uses an intelligent genetic algorithm with an inheritable mechanism (IGA) [31,32], combined with an SVM classifier, to simultaneously identify a small number $m$ out of a large number $n$ of GO terms as input features, where $m<<n$. The exploration of the $m$ informative GO terms from $n$ candidate GO terms is a combinatorial optimization problem $\mathrm{C}(n, m)$ with a huge search space of size $\mathrm{C}(n, m)=n ! /(m !(n-m) !))$. An IGA based on orthogonal experimental design using a divide-and-conquer strategy and systematic reasoning method can efficiently solve this large combinatorial optimization problem.

The leave-one-out cross-validation (LOOCV) is considered to be the most rigorous and objective test. Although bias-free, this test is very computationally demanding and is often impractical for large data sets. The $\mathrm{N}$-fold crossvalidation not only provides a bias-free estimation of the accuracy at a much reduced computational cost, but is also considered as an acceptable test for evaluating prediction performance of an algorithm [44]. Therefore, GOmining uses the prediction accuracy of $10-\mathrm{CV}$ as the fitness function to perform IGA on the entire training sets of proteins under considering the computation cost.

The input of the algorithm GOmining is composed of 1) a training set of protein sequences categorized into a number of compartments (classes), and 2) the essential GO terms corresponding to the compartments. The output comprises a set of $m$ informative GO terms and the associated parameter settings of an SVM classifier. Since the novel sequences without known accession numbers use BLAST to obtain annotated GO terms, all training sequences use the same BLAST to obtain GO terms for consistence.

Step 1: (preparation of SVM) The multi-classification problem is solved by using a series of binary classifiers of LIBSVM [39]. In this study, the kernel parameter $\gamma$ and cost parameter $C$ are tuned where $\gamma \in\left\{2^{-7}, 2^{-6}, \ldots, 2^{8}\right\}$ and $C \in\left\{2^{-7}, 2^{-6}, \ldots, 2^{8}\right\}$.

Step 2: (sequence representation) Obtain annotated GO terms from the GOA database for all training proteins using BLAST with $h=1$ and $e=10^{-9}$. Let $n$ be the total number of GO terms that appear among all proteins in the training data set. For example, $n=1714$ and $n=2870$ were derived for SCL12L and SCL16L, respectively. The protein is represented as an $n$-dimensional binary feature vector. 
Table I2: Distribution of the $m$ informative $G O$ terms. Most instructive $G O$ terms (80\%) are not offspring of the essential $G O$ terms that the ratios are $26 / 32$ and $36 / 45$ for SCLI $2 L$ and SCLI 6 L, respectively.

\begin{tabular}{|c|c|c|}
\hline & $\mathrm{SCL} \mid 2 \mathrm{~L}(m=44)$ & SCLI6L $(m=60)$ \\
\hline Essential GO terms & I2: I (B), II (C) & I5: I(B), I4(C) \\
\hline Instructive GO terms: & $32:$ & 45: \\
\hline (a) offspring of some essential GO term & $4(C)$ & $9(\mathrm{C})$ \\
\hline (b) between two essential GO terms & $2(\mathrm{C})$ & 0 \\
\hline (c) not offspring of any essential GO term & $7(\mathrm{M}), \mathrm{I} 4(\mathrm{~B}), 5(\mathrm{C})$ & I8(M), I3(B), 5(C) \\
\hline
\end{tabular}

Step 3: (inclusion of essential GO terms) Identify $d$ essential GO terms out of $n$ GO terms and number them from 1 to $d$. For example, $d=12$ and $d=15$ were found from SCL12L and SCL16L, respectively.

Step 4: (chromosome encoding) The IGA-chromosome comprises $n$ binary IGA-genes $f_{i}$ for selecting informative GO terms and two 4-bit IGA-genes for encoding $\gamma$ and $C$, where $f_{i}=1, i=1, \ldots, d$. The $i$ th GO term is included in the feature set of the SVM classifier if $f_{i}=1$; otherwise, the $i$ th GO term is excluded $\left(f_{i}=0\right)$. Figure 5 shows the sequence representation and IGA-chromosome encoding method.

Step 5: (initial solution) Perform IGA to select $r_{\text {start }}$ out of $n$ GO terms, i.e., the solution to $\mathrm{C}\left(n, r_{\text {start }}\right)$, where the $d$ GO terms are always selected. Table 13 shows the parameter settings of IGA, such as crossover probability $p_{c}=0.8$. The procedure of IGA is described in detail in the work [18].

Step 6: (inheritance mechanism) The inheritance mechanism of IGA can efficiently search for the solution to $\mathrm{C}(n$, $r+1)$ by inheriting a good solution $S_{r}$ to $\mathrm{C}(n, r)$. Obtain all solutions $S_{r}$ from $r=r_{\text {start }}+1, \ldots, r_{\text {end }}$ one by one using IGA $[31,32]$. For example, $r_{\text {start }}=40$ and $r_{\text {end }}=70$ according to former experience.

Step 7: (decoding chromosome) Let $S_{m}$ be the most accurate solution with $m$ selected GO terms among all solutions $S_{r}$. Obtain the $m$ informative GO terms and parameter values of $\gamma$ and $C$.

Step 8: (robust performance) Perform Steps 5-7 for $N$ independent runs to obtain the best one of $N$ solutions $S_{m}$ and the associated parameter settings of the SVM parameters. The best solution considers both high prediction accuracy and high mean frequency of the $m$ selected GO terms appeared in the $N$ runs. In this study, $N=30$.

\section{ProLoc-GO}

As shown in Fig. 6, each query protein is first BLASTed with $h=1$ and $e=10^{-9}$ against the Swiss-Prot database to obtain a homology with a known accession number. If no such homology exists, then adjust the threshold value $e$ of BLAST until the desired homology is obtained, where $h=$
1 and $e \in\left\{10^{-9}, 10^{-8}, \ldots, 10^{-1}\right\}$. The accession number of the homology of each protein sequence in SCL12 and SCL1 6 was obtained by using BLAST with $h=1$ and $e=10^{-}$ 9 . This accession number is used as input to the GOA database for retrieving the corresponding $k(>1)$ GO terms: $\mathrm{GO}: 1, \mathrm{GO}: 2, \ldots \mathrm{GO}: \mathrm{k}$. If none of the $k \mathrm{GO}$ terms belongs to the set of $m$ informative GO terms, then the sequence is represented using an $n$-dimensional binary vector and is predicted by the SVM-GO classifier. Otherwise, the sequence is represented as an $m$-dimensional binary vector and is predicted by the SVM-IGO classifier. Notably, the SVM-GO classifier predicts only a very small percentage of input sequences. ProLoc-GO is derived from the two major classifiers SVM-GO and SVM-IGO for subcellular localization prediction.

\section{Fuzzy k-NN}

The protein is represented as an $n$-dimensional binary vector and the generalized distance between two proteins $\mathbf{P}$ and $P_{i}[2]$ is denoted as :

$$
D\left(\mathbf{P}, \mathbf{P}_{i}\right)=1-\mathbf{P} \cdot \mathbf{P}_{i} /\|\mathbf{P}\|\left\|\mathbf{P}_{i}\right\|
$$

where $\mathbf{P} \cdot \mathbf{P}_{i}$ is the dot product of vectors $\mathbf{P}$ and $\mathbf{P}_{i}$, and $\|\mathbf{P}\|$ and $\left\|\mathbf{P}_{i}\right\|$ are their moduli.

This study determined the best value of $k$ by using a stepwise approach where $k \in\{1,2, \ldots, 10\}$.

The fuzzy $k-\mathrm{NN}$ classifier $[13,29,30]$ is a variation of $k-\mathrm{NN}$, which assign fuzzy membership values $r_{c}(\mathbf{P})$ of a query sequence $\mathbf{P}$ to each class $c$ as follows:

$$
r_{c}(\mathbf{P})=\frac{\sum_{j=1}^{k} r_{c}\left(\mathbf{P}^{j}\right)\left|\mathbf{P}-\mathbf{P}^{j}\right|^{-2 /(w-1)}}{\sum_{j=1}^{k}\left|\mathbf{P}-\mathbf{P}^{j}\right|^{-2(w-1)}}, c=1,2, \ldots, N_{C^{\prime}}
$$

where the distance is calculated by according to (1). In this study, the best values of parameters $(k, w)$ are tuned iteratively from $k \in\{1,2, \ldots, 10\}$ and $w \in\{1.05,1.10, \ldots$, $1.95\}$ for the fuzzy $k-N N$ classifier. 


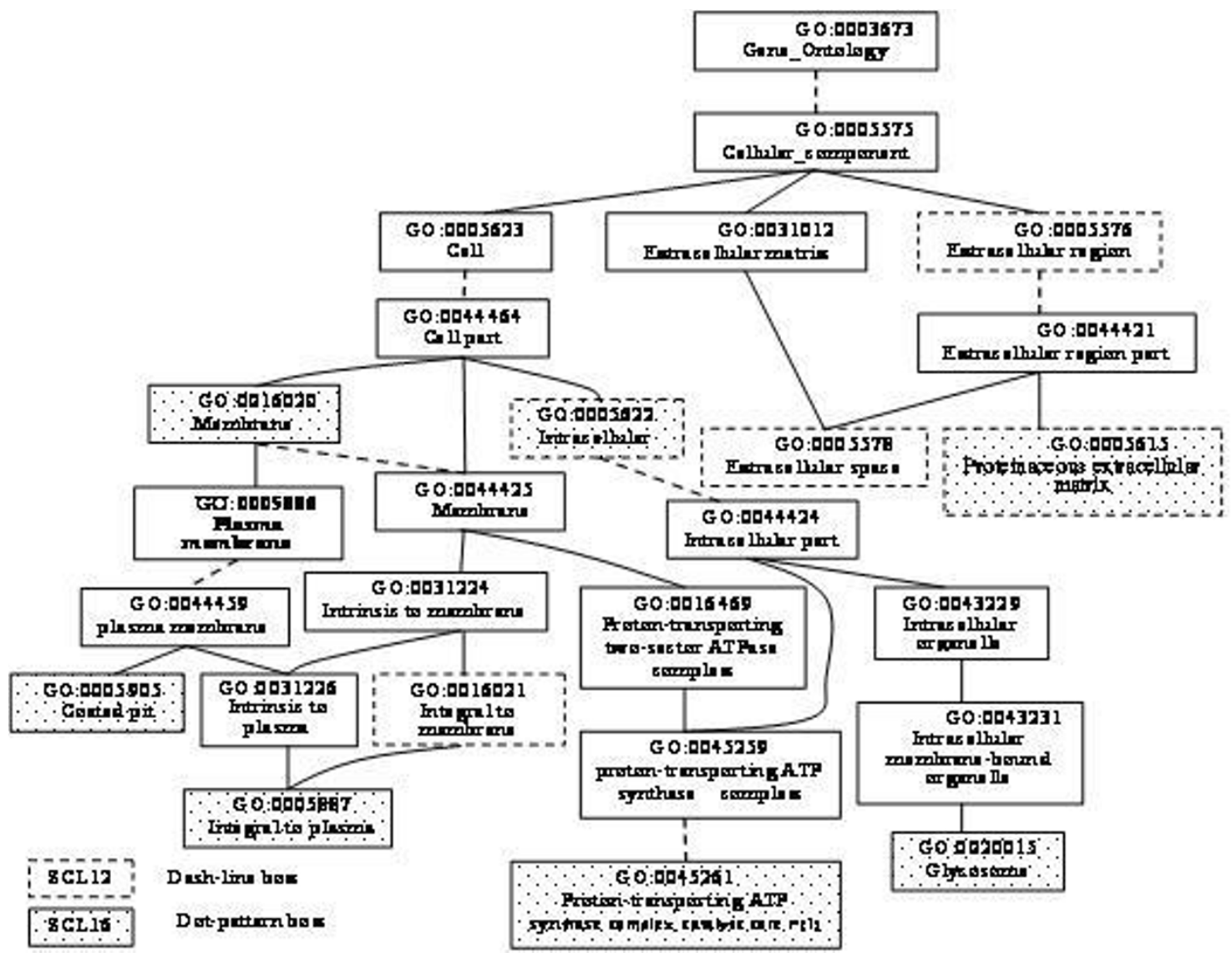

Figure 4

Some of the selected GO terms are NOT offspring of any essential GO terms. For SCLI $2 \mathrm{~L}$, five instructive GO terms are shown belonging to cellular component branch: GO:00 I602I, GO:0005576, GO:0005622, GO:0005578 and GO:00056 15, which are not offspring of essential GO terms. For SCLI6L, five GO terms belonging to the class (c) are shown: GO:0005622, GO:00056I5, GO:00200I5, GO:0016020 and GO:004526I. GO:0005905 and GO:0005887 belong to the class (a).

\section{SVM-RBS}

To evaluate the proposed IGA-based feature selection method GOmining, this study implements a classifier SVM-RBS by using SVM with a subset of the $n$ GO terms by the rank-based selection (RBS) method [17,42]. One previous work on ProLoc [18] showed that this univariate method RBS is inferior to the multivariate feature selection by IGA for selecting physicochemical properties. First, each of all $n$ GO terms (for example, $n=1714$ for SCL12L) is ranked according to the accuracy of SVM with the evaluated single feature, where the best values of parameters $(C, \gamma)$ were determined using a step-wise approach where $\gamma \in\left\{2^{-7}, 2^{-6}, \ldots, 2^{8}\right\}$ and $C \in\left\{2^{-7}, 2^{-6}, \ldots\right.$, $\left.2^{8}\right\}$. The top-ranking 70 features $a_{\mathrm{i}}, i=1, \ldots, 70$ are then picked, and the top-ranking 40 features with $r=40$ are used as an initial feature set $\left\{b_{1}, \ldots, b_{40}\right\}$. Consequently, the feature set with size $r+1$ is incrementally established by adding the best feature $b_{r+1}$ (having the highest accu-

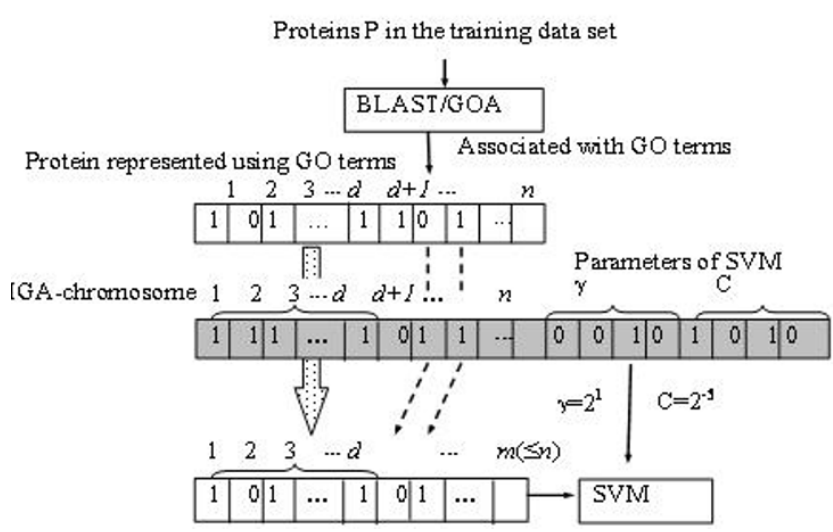

Figure 5

Sequence representation and IGA-chromosome encoding method. 
Table 13: The used control parameters of IGA

\begin{tabular}{lc}
\hline Parameter & Value \\
\hline Population size $N_{\text {pop }}$ & 50 \\
Selection probability $P_{\mathrm{s}}$ & 0.2 \\
Crossover probability $P_{\mathrm{c}}$ & 0.8 \\
Mutation probability $P_{\mathrm{m}}$ & 0.05 \\
Factor number of orthogonal arrays & 7 \\
Maximum generations $G_{\max }$ & 60
\end{tabular}

racy of SVM using $10-\mathrm{CV}$ ) from the remaining 70-r features into the current feature set.

\section{Authors' contributions}

WLH designed the system, implemented programs, participated in manuscript preparation and carried out the detail study. CWT designed the system and implemented programs. SWH, SFH and SYH conceived the idea of this work. Additionally, SYH supervised the whole project and participated in manuscript preparation. All authors have read and approved the final manuscript.

\section{Availability}

The prediction system using ProLoc-GO with input sequences of query proteins for protein subcellular locali-

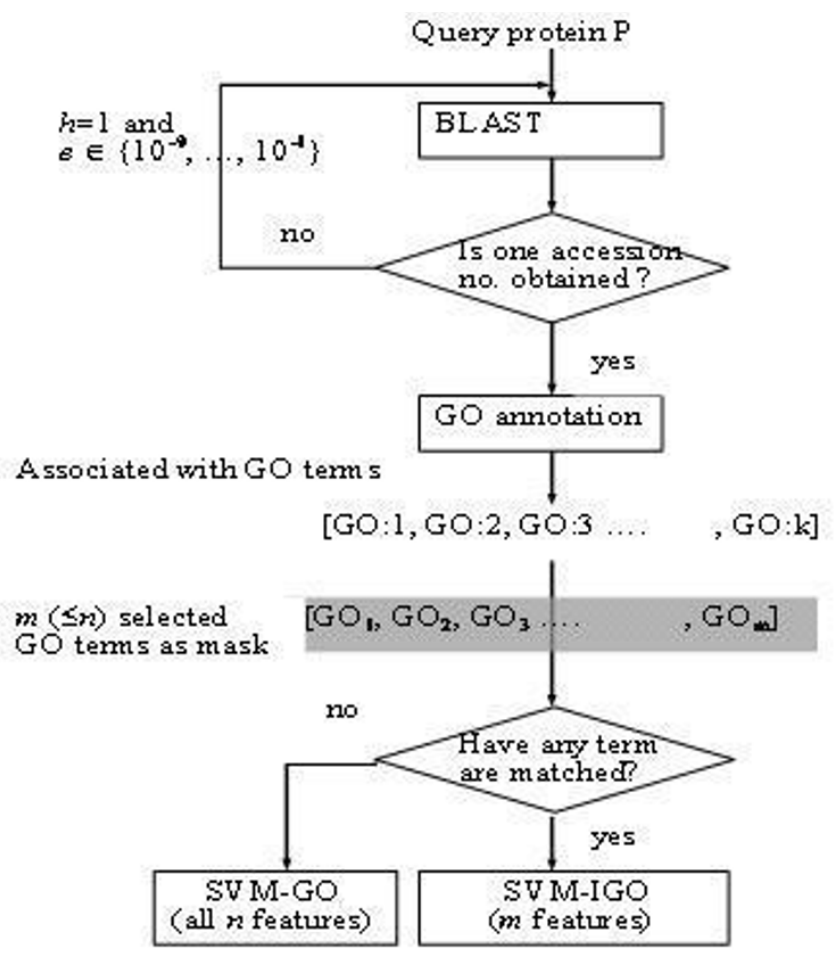

Figure 6

Prediction flowchart of ProLoc-GO using both classifiers SVM-IGO and SVM-GO. zation has been implemented at http:// iclab.life.nctu.edu.tw/prolocgo.

\section{Acknowledgements}

The authors would like to thank the National Science Council of Taiwan for financially supporting this research under the contract numbers NSC 96-

2628-E-009-I4I-MY3 and NSC 96-2627-B-009-002.

\section{References}

I. Ashburner M, Ball CA, Blake JA, Botstein D, Butler H, Cherry JM, Davis AP, Dolinski K, Dwight SS, Eppig JT, Harris MA, Hill DP, IsselTarver L, Kasarskis A, Lewis S, Matese JC, Richardson JE, Ringwald M, Rubin GM, Sherlock G: Gene ontology: tool for the unification of biology. The Gene Ontology Consortium. Nat Genet 2000:25-29.

2. Chou KC, Shen HB: Predicting Eukaryotic Protein Subcellular Location by Fusing Optimized Evidence-Theoretic K-Nearest Neighbor Classifiers. J Proteome Res 2006, 5(8): | 888 - I | 897.

3. Chou KC, Shen HB: Euk-mPLoc: A Fusion Classifier for LargeScale Eukaryotic Protein Subcellular Location Prediction by Incorporating Multiple Sites. J Proteome Res 2007, 6(5): I728-34. Epub $2007 \quad$ Mar 31.

4. Chou KC, Shen HB: Hum-PLoc: A novel ensemble classifier for predicting human protein subcellular localization. Biochem Biophys Res Commun 2006, 347:150-157.

5. Lei Z, Dai Y: Assessing protein similarity with Gene Ontology and its use in subnuclear localization prediction. BMC Bioinformatics 2006:49I-590.

6. Emanuelsson $\mathrm{O}$, Nielsen $\mathrm{H}$, Brunak $\mathrm{S}$, von Heijne G: Predicting Subcellular Localization of Proteins Based on their N-terminal Amino Acid Sequence. J Mol Biol 2000, 300(4): 1005- 1016.

7. Nakai K, Horton P: PSORT: a program for detecting sorting signals in proteins and predicting their subcellular localization. Trends Biochem Sci 1999, 24:34-35.

8. Cedano J, Aloy P, P'erez-Pons JA, Querol E: Relation between amino acid composition and cellular location of proteins. J Mol Biol 1997, 266:594-600.

9. Nakashima $\mathrm{H}$, Nishikawa K: Discrimination of intracellular and extracellular proteins using amino acid composition and residue-pair frequencies. I Mol Biol I994, 238:54-6I.

10. Park KJ, Kanehisa M: Prediction of protein subcellular locations by support vector machines using compositions of amino acid and amino acid pairs. Bioinformatics 2003, 19:1656-1663.

II. Garg A, Bhasin M, Raghava GP: Support vector machine-based method for subcellular localization of human proteins using amino acid compositions, their order, and similarity search. J Biol Chem 2005, 280: I 4427-I4432.

12. Hua S, Sun Z: Support vector machine approach for protein subcellular localization prediction. Bioinformatics 200I, I 7:72I-728

13. Huang Y, Li Y: Prediction of protein subcellular locations using fuzzy k-NN method. Bioinformatics 2004, 20:2I-28.

14. Yu CS, Lin CJ, Hwang JK: Predicting subcellular localization of proteins for Gram-negative bacteria by support vector machines based on n-peptide compositions. Protein Sci 2004, I3:| 402-| 406.

15. Bhasin M, Garg A, Raghava GPS: PSLpred: prediction of subcellular localization of bacterial proteins. Bioinformatics 2005, 21:2522-2524.

16. Bhasin M, Raghava GPS: ESLpred: SVM-based method for subcellular localization of eukaryotic proteins using dipeptide composition and PSI-BLAST. Nucleic Acids Res 2004 32:W4I4-4I9.

17. Sarda D, Chua G, Li KB, Krishnan A: pSLIP: SVM based protein subcellular localization prediction using multiple physicochemical properties. BMC Bioinformatics 2005, 6:152-163.

18. Huang WL, Tung CW, Huang HL, Hwang SF, Ho SY: ProLoc: Prediction of protein subnuclear localization using SVM with automatic selection from physicochemical composition features. BioSystems 2007, 90(2):573-581.

19. Nanni L, Lumini A: An ensemble of K-local hyperplanes for predicting protein-protein interactions. Bioinformatics 2006 , 22: $1207-1210$. 
20. Cai YD, Liu XJ, Xu XB, Chou KC: Support vector machines for prediction of protein subcellular location by incorporating quasi-sequence-order effect. J Cell Biochem 2002, 84:343-348.

21. Chou KC, Shen HB: Predicting protein subnuclear location with optimized evidence-theoretic K-nearest classifier and pseudo amino acid composition. Biochem Biophys Res Commun 2005, 337:752-756.

22. http://www.geneontology.org/.

23. Yi G, Sze SH, Thon MR: Identifying clusters of functionally related genes in genomes. Bioinformatics, 2007, 23(9):1053-60. Epub 2007 Jan 19.

24. Lewin A, Grieve I: Grouping Gene Ontology terms to improve the assessment of gene set enrichment in microarray data. BMC Bioinformatics 2006, 7(I):426.

25. Carroll S, Pavlovic V: Protein classification using probabilistic chain graphs and the Gene Ontology structure. Bioinformatics 2006, 22(I5): 187|-I878.

26. Wolstencroft K, Lord P, Tabernero L, Brass A, Stevens R: Protein classification using ontology classification. Bioinformatics 2006, 22(I4): $530-538$

27. Qian Z, Cai YD, Li Y: A novel computational method to predict transcription factor DNA binding preference. Biochem Biophys Res Commun 2006, 348(3): I034-1037.

28. Popescu M, Keller JM, Mitchell JA: Fuzzy Measures on the Gene Ontology for Gene Product Similarity. IEEE/ACM Trans Comput Biol Bioinformatics 2006, 3(3):263-74.

29. Huang WL, Chen HM, Hwang SF, Ho SY: Accurate prediction of enzyme subfamily class using an adaptive fuzzy k-nearest neighbor method. BioSystems, 2007, 90(2):405-4I8. Epub 2006 Oct 26.

30. Keller JM, Gray MR, Givens JA: A fuzzy k-nearest neighbours algorithm. IEEE Trans Syst Man Cybern 1985, I5:580-585.

31. Ho SY, Chen JH, Huang MH: Inheritable genetic algorithm for biobjective $0 / \mathrm{I}$ combinatorial optimization problems and its applications. Systems, Man and Cybernetics, Part B, IEEE Transactions on 2004, 34(I):609-620.

32. Ho SY, Shu LS, Chen JH: Intelligent evolutionary algorithms for large parameter optimization problems. Evolutionary Computation, IEEE Transactions on 2004, 8:522-54I.

33. Apweiler R, Bairoch A, Wu CH, Barker WC, Boeckmann B, Ferro S, Gasteiger E, Huang H, Lopez R, Magrane M, Martin MJ, Natale DA, O'Donovan C, Redaschi N, Yeh LS: UniProt: the Universal Protein knowledgebase. Nucleic Acids Res 2004, 32:DI I5-DII 9.

34. Wang GL, Dunbrack Jr. RL: PISCES: a protein sequence culling server. Bioinformatics 2003, 19:|589-|59|.

35. Camon E, Magrane M, Barrell D, Lee V, Dimmer E, Maslen J, Binns D, Harte N, Lopez R, Apweiler R: The Gene Ontology Annotation (GOA) Database: sharing knowledge in Uniprot with Gene Ontology. Nucleic Acids Res 2004, 32(Database issue):D262-266.

36. GOA. [ftp://ftp.ebi.ac.uk/pub/databases/GO/goa/UNIPROT/ ].

37. Altschul SF, Gish W, Miller W, Myers EW, Lipman DJ: Basic local alignment search tool. I Mol Biol 1990, 21 5(3):403-4I0.

38. Altschul SF, Madden TL, Schaffer AA, Zhang J, Zhang Z, Miller W, Lipman DJ: Gapped BLAST and PSIBLAST:a new generation of protein database search programs. Nucleic Acids Res 1997, 25:3389-3402.

39. Chang CC, Lin CJ: LIBSVM : a library for support vector machines, Software available at http://www.csie.ntu.edu.tw/ $\sim$ cjlin/libsvm. 200I.

40. Ho SY, Hsieh $\mathrm{CH}$, Chen HM, Huang HL: Interpretable gene expression classifier with an accurate and compact fuzzy rule base for microarray data analysis. BioSystems 2006 85:165-176.

41. Tung CW, Ho SY: POPI: Predicting immunogenicity of MHC class I binding peptides by mining informative physicochemical properties. Bioinformatics 2007, 23(8):942-949.

42. Li T, Zhang C, Ogihara M: A comparative study of feature selection and multiclass classification methods for tissue classification based on gene expression. Bioinformatics 2004 20:2429-2437.

43. Lu Z, Hunter L: GO Molecular Function Terms Are Predictive of Subcellular Localization. Pac Symp Biocomput 2005: I5 I-16 I.

44. Stone M: Cross-validatory choice and assessment of statistica predictions. Journal of the Royal Statistical Society 1974, 36: I I I- I47.
Publish with Bio Med Central and every scientist can read your work free of charge

"BioMed Central will be the most significant development for disseminating the results of biomedical research in our lifetime. "

Sir Paul Nurse, Cancer Research UK

Your research papers will be:

- available free of charge to the entire biomedical community

- peer reviewed and published immediately upon acceptance

- cited in PubMed and archived on PubMed Central

- yours - you keep the copyright 